\title{
About A New Mastopexy Augmentation Method Using Inferior Flap and Pectoral Muscle Loop in Wise Pattern Mammaplasty
}

\section{Abdolazim Ghalambor, Kamran Babaei, Arasteh Torabi Farsani*}

Department of Plastic and Reconstructive Surgery, Ahvaz Jundishapur University of Medical Sciences, Ahvaz, Iran

Study Area: Ahvaz, Iran

Coordinates: $31^{\circ} 19^{\prime} 13^{\prime \prime N} ; 48^{\circ} 40^{\prime} 09^{\prime \prime E}$

Keywords: Scars, Nipple-areola, Breast size

Ethical Code: Approved by the ethics committee of the Ahvaz Jundishapur University of Medical Sciences (Code of Ethics: IR.AJUMS.REC.1397.687)

\section{Introduction:}

Reduction mammaplasty is one of the most commonly used plastic surgical procedures (Anastasatos, 2016; Villani et al., 2009). In most parts of the world, with the increment in the number of such surgery the reduction in breast mammaplasty is rapidly becoming one of the most commonly used plastic surgeries (Daane \& Rockwell, 1999).

A desirable reduction mammaplasty for breast size should be accompanied by appropriate size and shape, minimal scar, natural nipple feeling, and lactation ability. Also, this procedure should be simple, fast, with the least complication, minimal bleeding during surgery, and possible to be done by surgeons (Cerovac et al., 2005; Kneser et al., 2005). The purpose of such surgery is to minimize and extend the breast as much as desired, with good looks, longlasting results, reduced surgical scars, and low-tech technique (Abramson et al., 2005; Rohrich et al., 2004 ). So far, more than 100 different techniques have been mentioned for this operation (Daane et al., 1999; Rohrich et

\section{$\underline{\text { Abstract }}$}

Developing new surgical techniques leaves scars which are cosmetically acceptable, reducing the need for subsequent surgical procedures. The present study aimed to investigate the outcomes of a new auto-augmentation method using inferior flap and pectoral muscle loop in wise pattern mammaplasty methods. It was done by a retrospective study based on the medical records of patients $(n=26$; age $=20$ years old) with Regnault ptosis grade II or III who recently underwent inferior mammaplasty surgery. Following the demographic information of the patients, the inclusion and exclusion criteria of the study were extracted and the eligible patients were contacted to attend the clinic for assessments of the surgery outcomes. The assessment factors were nipple-areola pain, post-operation sternal notch-nipple distance, inframammary fold-nipple distance up to the inferior nipple, breast symmetry on both sides, loss of nipple-areola, requesting re-surgery, overall patient satisfaction, and complications. The average satisfaction score was 3.27 out of 4. Complications included one case of seroma, one ulcer opening and one case of nipple-areola necrosis. Finally, the new augmentation method using inferior flap and pectoral muscle loop could be suggested as a suitable technique for reduction mammoplasty having low complications with high satisfaction in the patient.

al., 2004). In patients having severe enlargement and ptosis, breast surgery should be recommended through the inferior flap. The deformation and significant reduction of breast size are the advantages of this technique (Warren \& Neligan, 2012). If a person, in addition to the restoration of the size of the breasts, wishes to have the breasts at a higher level, a mastopexy surgery should be preferred. In this type of surgery, surgeons seek a method with the least complication and long-term stability. Breast lift surgery incisions are performed in a variety of ways, including Dounut, Lolly Pop, Keyhole, Anchor, and Wise pattern. The Wise pattern method is used in women who have a severe drop in the breast and tip. They cannot achieve the desired results in other ways. In this study, the surgery was done through the Wise pattern method (Warren \& Neligan, 2012; Lalonde et al., 2003; Swanson, 2011). The purpose of mastopexy is a breast lift is usually for a more rounded shape, nipple lift, and reducing the nipple-areola size (Mofid et al., 2002; Schlenz et al., 2005). According to the 
above, the purpose of this study was to investigate a new method for filling the upper fissure and reducing the incidence of post-operativeptosis. Therefore, this study aimed to investigate the use of inferior flap and pectoral muscle loop as a new auto-augmentation method in the Wise pattern mammaplasty methods to help achieve a method with the highest patient satisfaction, the least complications, and long-term stability.

\section{Methodology:}

This was a retrospective study based on hospital information in 2017-2018 on 26 patients older than 20 years with grade II and III ptosis (according to Regnault classification) who referred to Taleghani Hospital of Ahwazand underwent reduction mammaplasty during 2017-2018. The inclusion criteria of the study included patients with bulky breast and candidate for reduction, health of the breasts and the armpit area for the presence of the mass, smoking cessation from a month before surgery in smokers and at least two weeks after surgery, passing at least 6 months from breastfeeding and lack of breast milk, and patients requiring resection of more than 600 grams. The patients, who elapsed at least one year since surgery were included. Patients with very large breasts and candidate for free nipple graft, history of mammaplasty, history of mastectomy, breast cancer patients, presence of scar in the chest and breast, patients with definite history of colloid formation in the wound, patients with radiotherapy history in the chest, and patients with a history of malignant disease were excluded. All patients over 35 years were examined for chest sonography and mammography prior to surgery and were excluded from the study in case of a defect in the case.

In the patients under study, the main pedicle was superomedial and in all patients, a 5 to $10-\mathrm{cm}$ flap was maintained in the center of the meridian line of the chest, which was used for the upper bridge auto-augmentation and was driven under the pectoral muscle. All patients in the study were operated by a surgeon and a surgical team. Following the inclusion and exclusion criteria of the study, the demographic information of patients, including age (year), body mass index ( $\mathrm{kg} / \mathrm{m} 2)$, marital status and history of lactation, eligible patients were contacted to attend the clinic were extracted. Proper consents were taken from the patients whowere under clinical examination.

The parameters for evaluating the treatment outcomes were nipple-areola pain, post-operation sternal notch-nipple (SN) distance $(\mathrm{cm})$, inframammary fold-nipple (IMF) distance up to the inferior nipple $(\mathrm{cm})$, breast symmetry on both sides, loss of nipple-areola, requesting re-surgery, overall patient satisfaction, and complications (hematoma, wound opening, seroma, and infection). To determine the patients' satisfaction with surgery, 7 questions were asked in the form of a pre-designed checklist in the last follow-up and, based on that, their overall satisfaction was also assessed as scores ranging from 1 to 4 (1: dissatisfied, 2: neutral, 3: satisfied, 4: extremely satisfied). The collected data were analyzed using SPSS 22 software, and the signif icance level was less than 0.05 .

\section{Result:}

The demographic characteristics of the Experimental and Control groups (EG, CG) are shown in Table-1.

Table-1: Demographic Characteristics of the subjects in groups

\begin{tabular}{lll}
\hline Variables & Mean \pm SD/ & N (\%) \\
\hline Age (year) & $46.21 \pm 12.07$ & $(27-61)$ \\
Follow up (month) & $14.68 \pm 3.59$ & $(12-21)$ \\
BMI (kg/m2) & $26.84 \pm 3.41$ & \\
$\quad$ Grade II & $15(57.69 \%)$ & \\
$\quad$ Ptosis III & $11(42.31 \%)$ & \\
Marital Status & \\
$\quad$ Married & $18(69.23 \%)$ & \\
$\quad$ Single & $8(30.77 \%)$ & \\
Breastfeeding experience & \\
$\quad$ Yes & $20(76.92 \%)$ \\
$\quad$ No & $6(23.08 \%)$ \\
\hline
\end{tabular}

A total of $17(65.38 \%)$ patients reported as complete satisfaction with nipple-areola feeling, 8 (30.77\%) patients expressed nipple-areola feeling compared to pre-operative as "moderate", and the nipple-areola feeling was weak in 1 $(3.85 \%)$ patient. Only one patient was dissatisfied with the nipple-areola feeling. The distance between sternal notch to the nipple and the distance between the IMF to the inferior nipple after the operation were evaluated in the last followup, the results of which are shown in Table-2.

Table-2: Distance between SN and IMF after the operation and in the last follow-up

\begin{tabular}{lll}
\hline Variables & Mean \pm SD/ & N (\%) \\
\hline Sternal Notch- & After surgery & $34.65 \pm 3.09$ \\
Nippledistance $(\mathbf{c m})$ & Latest follow up & $36.07 \pm 3.52$ \\
Infra mammary Fold- & After surgery & $7.39 \pm 2.69$ \\
Nippledistance $(\mathbf{c m})$ & Latest follow up & $8.12 \pm 2.15$ \\
\hline
\end{tabular}

In general, 22 patients $(84.62 \%)$ were "satisfied" or "very satisf ied" with the treatment. Only one patient, due to a lack of satisfaction with the nipple-areola position, requested for surgical revision and raised the nipple-areola position. Also, the mean score of patients' satisfaction was calculated to be 3.27 out of 4 (Table-3).

Complications were in $1(3.85 \%)$ case of seroma and 1 (3.85\%) case of ulcer opening. Nipple-areola necrosis was also observed in 1 case $(85.5 \%)$, which improved after three weeks with medication care and treatment. No cases of infection or hematoma were reported. There were also no cases of deformity in the breast and recurrence of ptosis.

\section{Discussion:}

In the present study, the majority of patients (65.38\%) were satisfied with nipple-areola feeling and 30.77\% expressed nipple-areola feeling compared to pre-operative as "moderate". Only one patient was dissatisfied with the 
ORIGINAL ARTICLE

Table-3: Overall satisfaction of patients according to the questionnaire

\begin{tabular}{lll}
\hline Patient satisfaction & No.of patients (\%) & \\
\hline Size of Breasts & 2 & $2(7.69 \%)$ \\
& 3 & $13(50 \%)$ \\
Amount of lift achieved & 4 & $11(42.31 \%)$ \\
& 3 & $1(3.85 \%)$ \\
Scar quality & 4 & $12(46.15 \%)$ \\
& 2 & $13(50 \%)$ \\
Nipple/areola position & 1 & $3(11.54 \%)$ \\
& 2 & $10(38.46 \%)$ \\
& 4 & $13(50 \%)$ \\
Nipple/areola size & 3 & $1(3.85 \%)$ \\
& 4 & $2(7.69 \%)$ \\
& 2 & $11(42.31 \%)$ \\
Softness/feel of breasts & 2 & $12(46.15 \%)$ \\
& 3 & $2(7.69 \%)$ \\
& 4 & $12(46.15 \%)$ \\
Achieving desired goals & 2 & $12(46.15 \%)$ \\
& 3 & $3(11.54 \%)$ \\
Overall Result & 4 & $11(42.31 \%)$ \\
& Dissatisfied & $12(46.15 \%)$ \\
& Neutral & $2(7.69 \%)$ \\
& Satisfied & $13(50 \%)$ \\
& Extremely satisfied & $11(42.31 \%)$ \\
\hline
\end{tabular}

nipple-areola feeling. The average satisfaction score of patients was 3.27 out of 4 . Complications included one case of seroma and one ulcer opening. The nipple-areola necrosis was also observed in one case that improved with medication care after three weeks. No cases of infection or hematoma were reported. There were also no cases of deformity in the breast and recurrence of ptosis. Soares et al. (2011), explained a mastopexy method called mastopexy with breast implants and flap pectoral muscle in 20 women with grade I and II mammary ptosis was evaluated. The duration of the follow-up was 9 to 12 months, during which there was no deformity in the breast and recurrence of ptosis. All surgically operated breast had a good shape and was well-f illed with a good bridge over the long term, and all patients were satisfied with the results of the treatment. Finally, it was concluded that mastopexy using breast implants and flap pectoral muscle is an easy technique to carry out, and has satisfactory results for a long time. Auersvald et al. (2010), a study on 22 patients underwent mastopexy with Regnault ptosis grade II or III and were augmented by pectoral muscle loop. The results of the evaluations showed a high level of satisfaction with treatment in patients. Finally, they concluded that mastopexy augmentation using a loop of the pectoral muscle to hold the implant as a new technique could provide long-lasting satisfactory results with the shape of the full upper breast bridge. In a study by Spear et al. (2004), the treatment results of 34 women undergoing a singlestage mastopexy and augmentation surgery in terms of patients and surgeons were evaluated. Subjects were interviewed by a questionnaire on satisfaction with treatment. The result they found was of mixed type on satisfaction level. Although the results of the treatment were reported to be well and were not excellent, and patients were not completely satisfied with the surgery, which could be partly due to the surgeon's skill or patient's personal taste. In our study, researchers suggested one-step mastopexy and breast augmentation surgery to be better than doing it in two separate steps.

Based on the results of the treatment, the use of the inferior flap and pectoral muscle loop as a new method of auto-augmentation could be referred as a suitable technique for reduction mammaplasty with low complications and long-term satisfactory results for the patient and surgeon.

\section{Acknowledgment:}

This study was approved in Ahvaz Jundishapur University of Medical Sciences (AJUMS). The study was financially supported by AJUMS (Grant.No.:U-97177).

\section{References:}

Abramson, D.L., Pap, S., Shifteh, S. \& Glasberg, S.B. (2005): Improving long-term breast shape with the medial pedicle wise pattern breast reduction. Plast. Reconstr. Surg., 115(7):1937-1943.

Anastasatos, J. (2016): Medial Pedicle and Mastopexy Breast Reduction. E-Source: Medscape.

Auersvald, A. \& Auersvald, L.A. (2011): Breast augmentation and mastopexy using a pectoral muscle loop. Aesthetic Plast. Surg., 35(3):333-340.

Cerovac, S., Ali, F.S., Blizard, R., Lloyd, G. \& Butler, P.E. (2005): Psychosexual function in women who have undergone reduction mammaplasty. Plast. Reconstr. Surg., 116(5):13o61313 .

Daane, S.P. \& Rockwell, W.B. (1999): Breast reduction techniques and outcomes: A meta-analysis. Aesthetic Surg. I., 19(4):293303.

Graf, R.M., Auersvald, A., Bernardes, A. \& Biggs, T.M. (200o): Reduction mammaplasty and mastopexy with shorter scar and better shape. Aesthetic Surg. I., 20(2):99-106.

Kneser, U., Jaeger, K., Bach, A.D., Polykandriotis, E., Ohnolz, J., Kopp, J. \& Horch, R.E. (2004): Breast-reduction surgery--a long-term survey of indications and outcomes. MMW Fortschr. Med., 146(42):36-38.

Lalonde, D.H., Lalonde, J. \& French, R. (2003): The no vertical scar breast reduction: a minor variation that allows to remove vertical scar portion of the inferior pedicle wise pattern $\mathrm{T}$ scar. Aesthetic Plast. Surg., 27(5):335-344.

Mofid, M.M., Dellon, A.L., Elias, J.J. \& Nahabedian, M.Y. (2002): Quantitation of breast sensibility following reduction mammaplasty: a comparison of inferior and medial pedicle techniques. Plast. Reconstr. Surg., 109(7):2283-2288. 
Qi, F., Gu, J., Zhang, X., Yang, Z., Shi, Y. \& Xu, J. (2004): Vertical reduction mammaplasty. Chinese J.Plast. Surg., 20(5):325-327.

Rohrich, R.J., Gosman, A.A., Brown, S.A., Tonadapu, P. \& Foster, B. (2004): Current preferences for breast reduction techniques: a survey of board-certified plastic surgeons 2002. Plast. Reconstr. Surg., 114(7):1724-1733.

Schlenz, I., Rigel, S., Schemper, M. \& Kuzbari, R. (2005): Alteration of nipple and areola sensitivity by reduction mammaplasty: a prospective comparison of five techniques. Plast. Reconstr. Surg., 115(3):743-751.

Soares, A.B., Franco, F.F., Rosim, E.T., Renó, B.A., Hachmann, J.O.P.A., Guidi, M.deC., Bueno, M.A.deC. \& Kharmandayan, P. (2011): Mastopexy with breast implants and the pectoralis major muscle flap: a technique adopted by the Department of Plastic Surgery of Unicamp. Rev. Bras. Cir. Plást., 26(4):659663.
Spear, S.L., Pelletiere, C.V. \& Menon, N. (2004): One-stage augmentation combined with mastopexy: aesthetic results and patient satisfaction. Aesthetic Plast. Surg., 28(5):259267.

Spector, D., Mayer, D.K., Knafl, K. \& Pusic, A. (2010): Not what I expected: informational needs of women undergoing breast surgery. Plast. Surg. Nurs., 30(2):70-74.

Swanson, E. (2011): A retrospective photometric study of 82 published reports of mastopexy and breast reduction. Plast. Reconstr. Surg., 128(6):1282-1301.

Villani, F., Caviggioli, F., Banzatti, B., Bandi, V. \& Maione L. (2009): Correlation between complication rate and perioperative riskfactors in superior pedicle reduction mammaplasty: our experience in 127 patients. Acta Chir. Plast., 51(3-4):65-68.

Warren, R.J. \& Neligan, P.C. (2012): Plastic Surgery-Aesthetic. Pub by: Elsevier Health Sciences. P. 924. 